\title{
Pan-FGFR Inhibitor LY2874455
}

National Cancer Institute

\section{Source}

National Cancer Institute. Pan-FGFR Inhibitor LY2874455. NCI Thesaurus. Code 139004.

An orally bioavailable pan-inhibitor of fibroblast growth factor receptor (FGFR) family proteins, with potential antineoplastic activity. Upon oral administration, FGFR inhibitor LY2874455 binds to and inhibits FGFR subtypes 1 (FGFR1), 2 (FGFR2), 3 (FGFR3) and 4 (FGFR4), which results in the inhibition of FGFR-mediated signal transduction pathways. This inhibits both tumor angiogenesis and proliferation of FGFR-overexpressing tumor cells. FGFR, a family of receptor tyrosine kinases upregulated in many tumor cell types, plays a key role in cellular proliferation, cell survival and angiogenesis. 